\title{
A new phenomenological investigation of KMR and MRW unintegrated parton distribution functions
}

\author{
M. Modarres ${ }^{1, \mathrm{a}}$, H. Hosseinkhani ${ }^{2}$, N. Olanj $^{3}$, M. R. Masouminia ${ }^{1}$ \\ ${ }^{1}$ Physics Department, University of Tehran, 1439955961 Tehran, Iran \\ 2 Plasma and Fusion Research School, Nuclear Science and Technology Research Institute, 14395-836 Tehran, Iran \\ ${ }^{3}$ Physics Department, Faculty of Science, Bu-Ali Sina University, 65178 Hamedan, Iran
}

Received: 22 August 2015 / Accepted: 19 November 2015 / Published online: 26 November 2015

(C) The Author(s) 2015. This article is published with open access at Springerlink.com

\begin{abstract}
We address the longitudinal proton structure function, $F_{L}\left(x, Q^{2}\right)$, from the $k_{t}$-factorization formalism by using the unintegrated parton distribution functions (UPDF) which are generated through the KMR and MRW procedures. The LO UPDF of the KMR prescription is extracted, by taking into account the PDF of Martin et al, i.e., MSTW2008LO and MRST99-NLO, and next the NLO UPDF of the MRW scheme is generated through the set of MSTW2008NLO PDF as the input. The different aspects of $F_{L}\left(x, Q^{2}\right)$ in the two approaches, as well as its perturbative and nonperturbative parts, are calculated. Then the comparison of $F_{L}\left(x, Q^{2}\right)$ is made with the data given by the ZEUS and $\mathrm{H} 1$ collaborations. It is demonstrated that the extracted $F_{L}\left(x, Q^{2}\right)$, based on the UPDF of two schemes, are consistent with the experimental data, and to a good approximation they are independent of the input PDF. But the one developed from the KMR prescription has better agreement with the data with respect to that of MRW. As has been suggested, by lowering the factorization scale or the Bjorken variable in the related experiments it may be possible to analyze the present theoretical approaches more accurately.
\end{abstract}

\section{Introduction}

In recent years, the extraction of unintegrated parton distribution functions (UPDFs) have become very important, since there exist plenty of experimental data on the various events, such as the exclusive and semi-inclusive processes in the high energy collisions in LHC, which indicate the necessity for a computation of these $k_{t}$-dependent parton distribution functions.

The UPDF, $f_{a}\left(x, k_{t}^{2}, \mu^{2}\right)$, are the two-scale dependent functions, i.e., $k_{t}^{2}$ and $\mu^{2}$, which satisfy the Ciafaloni-

\footnotetext{
a e-mail: mmodares@ut.ac.ir
}

Catani-Fiorani-Marchesini (CCFM) equations [1-5], where $x, k_{t}$, and $\mu$ are the longitudinal momentum fraction (the Bjorken variable), the transverse momentum, and the factorization scale, respectively. They are unintegrated over $k_{t}$ with respect to the conventional parton distribution functions (PDFs), which satisfy the Dokshitzer-Gribov-LipatovAltarelli-Parisi (DGLAP) evolution equations [6-9].

But the generation of UPDF from the CCFM equations is a complicated task. So, in general, the Monte Carlo event generators [10-17] are the main users of these equations. Since there is not a complete quark version of the CCFM formalism, the alternative prescriptions are used for producing the quark and the gluon UPDFs. Therefore, to obtain the UPDF, Kimber, Martin and Ryskin (KMR) [18,19] proposed a different procedure based on the standard DGLAP equations in the leading order (LO) approximation, along with a modification due to the angular ordering condition, which is the key dynamical property of the CCFM formalism. Later on, Martin, Ryskin and Watt (MRW) extended the KMR approach for the next-to-leading order (NLO) approximation [20-22], with the aim to improve the exclusive calculations. These two procedures are modifications to the standard DGLAP evolution equations and can produce the UPDF by using the PDF as the input.

The general behavior and stability of the KMR and MRW prescriptions were investigated in Refs. [24-28]. Furthermore, to check the reliability of the generated UPDF, their relative behaviors were compared and used to calculate the observable, deep inelastic scattering proton structure function $F_{2}\left(x, Q^{2}\right)$. Then the predictions of these two methods for the structure functions, $F_{2}\left(x, Q^{2}\right)$, were also compared to the electron-proton deep inelastic measurements of NMC [29], ZEUS [30], and H1+ZEUS [31] experimental data. The results were promising [32]. It is also concluded that [32], while the MRW formalism is more in compliance with the DGLAP evolution equations requisites, it seems that in 
the KMR case the angular ordering constraint spreads the UPDF to the whole transverse momentum region and makes the results sum up the leading DGLAP and Balitski-FadinKuraev-Lipatov (BFKL) logarithms [34-38].

Another important observable quantity in this connection is the longitudinal structure function, i.e., $F_{L}\left(x, \mu^{2}\right)$, which is proportional to the cross section of the longitudinal polarized virtual photon with proton. Particularly at small $x$, it is directly sensitive to the gluon distributions i.e. $g \rightarrow q \bar{q}$ process. Moreover, its calculations in this region need the $k_{t}$ factorization formalism [39-43], which is beyond the standard collinear factorization procedure [44]. Recently, Golec-Biernat and Staśto [45,46] (GS) have used the $k_{t}$ and collinear factorizations [39-43] as well as the dipole approach to generate the longitudinal structure function, but by using the DGLAP/BFKL re-summation method, developed by Kwiecinski, Martin and Stasto (KMS) [47], for a calculation of the unintegrated gluon density at small $x$. They have parameterized the input non-perturbative gluon distribution so that they could get the best fit to the experimental proton structure function data [47].

On the experimental side, the longitudinal structure function has been measured by both the $\mathrm{H} 1[48,49]$ and the ZEUS [50,51] collaborations at the DESY electron-proton collider HERA. The $Q^{2}$ ranges have been varied between 12-90 and 24-110 $\mathrm{GeV}^{2}$ in each experiment, respectively.

As was pointed out above, similar to our recent publication on $F_{2}\left(x, Q^{2}\right)$ [32], in the present paper, we intend to calculate $F_{L}\left(x, Q^{2}\right)$ by working in the $k_{t}$-factorization scheme. But rather than the KMS re-summation method pointed out above, the KMR and MRW [18-22] formalisms are used to predict the UPDF with the input PDF of the MRST99NLO [52], MSTW2008-LO [53] and MSTW2008-NLO [53], which covers a wide range of the $\left(x, Q^{2}\right)$ plane. Then our results can be compared both with the experimental data as well as the theoretical KMS-GS presentation of $F_{L}\left(x, Q^{2}\right)$. So the paper is organized as follows: in Sect. 2 we give a brief review of the KMR and the MRW formalisms [18-22] for the extraction of the UPDF form as regards the phenomenological PDF [52,53]. The formulation of $F_{L}\left(x, Q^{2}\right)$ based on the $k_{t}$-factorization scheme is given in Sect. 3. Finally, Sect. 4 is devoted to results, discussions, and conclusions.

\section{A brief review of the KMR and the MRW formalisms}

The KMR and MRW [18-23] ideas for generating the UPDF work as follows: Using the given integrated PDF as the inputs, the KMR and MRW procedures produce the UPDF as their outputs. They are based on the DGLAP equations along with some modifications due to the separation of virtual and real parts of the evolutions, and the choice of the splitting functions at leading order (LO) and the next-to-leading order (NLO) levels, respectively: (i) In the KMR formalism $[18,19]$, the UPDFs, $f_{a}\left(x, k_{t}^{2}, \mu^{2}\right)(a=q$ and $g)$, are defined in terms of the quarks and the gluons PDF, i.e.,

$$
\begin{aligned}
& f_{q}\left(x, k_{t}^{2}, \mu^{2}\right) \\
& =T_{q}\left(k_{t}, \mu\right) \frac{\alpha_{s}\left(k_{t}^{2}\right)}{2 \pi} \int_{x}^{1-\Delta} \mathrm{d} z\left[P_{q q}(z) \frac{x}{z} q\left(\frac{x}{z}, k_{t}^{2}\right)\right. \\
& \left.\quad+P_{q g}(z) \frac{x}{z} g\left(\frac{x}{z}, k_{t}^{2}\right)\right]
\end{aligned}
$$

and

$$
\begin{aligned}
& f_{g}\left(x, k_{t}^{2}, \mu^{2}\right) \\
& =T_{g}\left(k_{t}, \mu\right) \frac{\alpha_{s}\left(k_{t}^{2}\right)}{2 \pi} \int_{x}^{1-\Delta} \mathrm{d} z\left[\sum_{q} P_{g q}(z) \frac{x}{z} q\left(\frac{x}{z}, k_{t}^{2}\right)\right. \\
& \left.\quad+P_{g g}(z) \frac{x}{z} g\left(\frac{x}{z}, k_{t}^{2}\right)\right],
\end{aligned}
$$

respectively, where $P_{a a^{\prime}}(x)$ are the LO splitting functions, and the survival probability factors, $T_{a}\left(k_{t}, \mu\right)$, are evaluated from

$$
\begin{aligned}
& T_{a}\left(k_{t}, \mu\right) \\
& \quad=\exp \left[-\int_{k_{t}^{2}}^{\mu^{2}} \frac{\alpha_{s}\left(k_{t}^{\prime 2}\right)}{2 \pi} \frac{\mathrm{d} k_{t}^{\prime 2}}{k_{t}^{\prime 2}} \sum_{a^{\prime}} \int_{0}^{1-\Delta} \mathrm{d} z^{\prime} P_{a^{\prime} a}\left(z^{\prime}\right)\right]
\end{aligned}
$$

The angular ordering condition (AOC) [54,55], which is a consequence of coherent emission of gluons, on the last step of the evolution process [23] is imposed. The AOC determined the cut off, $\Delta=1-z_{\max }=\frac{k_{t}}{\mu+k_{t}}$, to prevent $z=1$ singularities in the splitting functions, which arises from the soft gluon emission. As has been pointed out in Refs. [18,19], the KMR approach has several main characteristics. The important one is the existence of the cut off at the upper limit of the integrals, which makes the distributions spread smoothly to the region in which $k_{t}>\mu$, i.e., the characteristic of small $x$ physics, which is principally governed by the BFKL evolution [34-38]. This feature of the KMR leads to the UPDF with the behavior very similar to the unified BFKL+DGLAP formalism [18,19]. The UPDFs based on the KMR formalism have been widely used in the phenomenological calculations which depend on the transverse momentum [17,56-66].

(ii) In the MRW formalism [20-22], a similar separation of real and virtual contributions to the DGLAP evolution is done, but the procedure is performed at the NLO level, i.e.,

$$
\begin{aligned}
f_{a}^{\mathrm{NLO}}\left(x, k_{t}^{2}, \mu^{2}\right) \\
=\int_{x}^{1} \mathrm{~d} z T_{a}\left(k^{2}, \mu^{2}\right) \frac{\alpha_{s}\left(k^{2}\right)}{2 \pi} \sum_{b=q, g} P_{a b}^{(0+1)}(z) b^{\mathrm{NLO}} \\
\quad \times\left(\frac{x}{z}, k^{2}\right) \Theta\left(\mu^{2}-k^{2}\right),
\end{aligned}
$$


where

$$
\begin{aligned}
& P_{a b}^{(0+1)}(z) \\
& \quad=P_{a b}^{(0)}(z)+\frac{\alpha_{s}}{2 \pi} P_{a b}^{(1)}(z), k^{2}=\frac{k_{t}^{2}}{1-z} .
\end{aligned}
$$

In Eqs. (4) and (5) the $P_{a b}^{(0)}$ and the $P_{a b}^{(1)}$ denote the LO and the NLO contributions of the splitting functions, respectively. It is obvious from Eq. (4) that, in the MRW formalism, the UPDFs are defined so as to ensure $k^{2}<\mu^{2}$. Also, the survival probability factors, $T_{a}\left(k^{2}, \mu^{2}\right)$, are obtained as follows:

$$
\begin{aligned}
T_{a}\left(k^{2}, \mu^{2}\right)= & \exp \left(-\int_{k^{2}}^{\mu^{2}} \frac{\alpha_{s}\left(\kappa^{2}\right)}{2 \pi} \frac{d \kappa^{2}}{\kappa^{2}}\right. \\
& \left.\times \sum_{b=q, g} \int_{0}^{1} d \zeta \zeta P_{b a}^{(0+1)}(\zeta)\right),
\end{aligned}
$$

where $P_{a b}^{(i)}$ (which is singular for $z \rightarrow 1$ ) is given in Ref. [67]. MRW have demonstrated that the sufficient accuracy can be obtained by keeping only the LO splitting functions together with the NLO integrated parton densities. So, by considering the angular ordering, we can use $P^{(0)}$ instead of $P^{(0+1)}$. As mentioned above, unlike the KMR formalism, where the angular ordering is imposed to all terms of Eqs. (1) and (2), in the MRW formalism, the angular ordering is imposed to the terms in which the splitting functions are singular, i.e. the terms that include $P_{q q}$ and $P_{g g}$.

\section{The formulation of $F_{L}\left(x, Q^{2}\right)$ in the $k_{t}$-factorization approach}

The $k_{t}$-factorization approach has been discussed in several works, i.e., Refs. [3,39,42,68,69]. In the following equation [45,70-72], the different terms, i.e., the perturbative and the non-perturbative contributions to the $F_{L}\left(x, Q^{2}\right)$ have been broken into the sum of gluons from the quark box (the first term, i.e., the $k_{t}$-factorization part), see Fig. 1 [22]), quarks (the second term), and the non-perturbative gluon (the third term) parts:

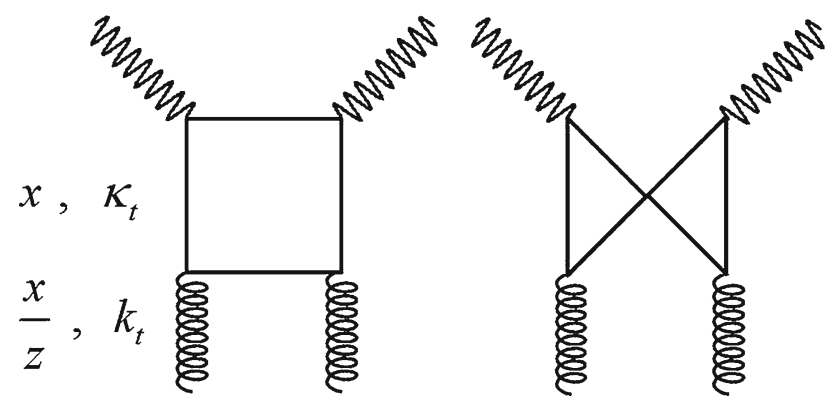

Fig. 1 The quark boxes and exchanged diagrams in the photon-gluon fusion process discussed in the $k_{t}$-factorization formula in the text

$$
\begin{aligned}
& F_{L}\left(x, Q^{2}\right) \\
& =\left[\frac{Q^{4}}{\pi^{2}} \sum_{q} e_{q}^{2} \int \frac{\mathrm{d} k_{t}^{2}}{k_{t}^{4}} \Theta\left(k^{2}-k_{0}^{2}\right) \int_{0}^{1} \mathrm{~d} \beta\right. \\
& \quad \times \int \mathrm{d}^{2} \kappa_{t} \alpha_{s}\left(\mu^{2}\right) \beta^{2}(1-\beta)^{2}\left(\frac{1}{D_{1}}-\frac{1}{D_{2}}\right)^{2} \\
& \left.\quad \times f_{g}\left(\frac{x}{z}, k_{t}^{2}, \mu^{2}\right) \Theta\left(1-\frac{x}{z}\right)\right] \\
& +\left[\frac{4}{3} \int_{x}^{1} \frac{\mathrm{d} y}{y} \frac{\alpha_{s}\left(Q^{2}\right)}{\pi}\left(\frac{x}{y}\right)^{2} F_{2}\left(y, Q^{2}\right)\right] \\
& +\frac{\alpha_{s}\left(Q^{2}\right)}{\pi}\left[\sum_{q} e_{q}^{2} \int_{x}^{1} \frac{\mathrm{d} y}{y}\left(\frac{x}{y}\right)^{2}\left(1-\frac{x}{y}\right) y g\left(y, k_{0}^{2}\right)\right],
\end{aligned}
$$

where the second term is (see $[73,74])$

$\sum_{q} e_{i}^{2} \frac{\alpha_{s}\left(Q^{2}\right)}{\pi} \frac{4}{3} \int_{x}^{1} \frac{\mathrm{d} y}{y}\left(\frac{x}{y}\right)^{2}\left[q_{i}\left(y, Q^{2}\right)+\bar{q}_{i}\left(y, Q^{2}\right)\right]$.

In the above equation, in which the graphical representations of $k_{t}$ and $\kappa_{t}$ have been introduced in Fig. 1, the variable $\beta$ is defined as the light-cone fraction of the photon momentum carried by the internal quark [69]. Also, the denominator factors are

$$
\begin{aligned}
& D_{1}=\kappa_{t}^{2}+\beta(1-\beta) Q^{2}+m_{q}^{2}, \\
& D_{2}=\left(\kappa_{\mathbf{t}}-\mathbf{k}_{\mathbf{t}}\right)^{2}+\beta(1-\beta) Q^{2}+m_{q}^{2} .
\end{aligned}
$$

Then by defining $\kappa_{\mathbf{t}}^{\prime}=\kappa_{\mathbf{t}}-(1-\beta) \mathbf{k}_{t}$, the variable $y$ takes the following form:

$$
y=x\left(1+\frac{\kappa^{\prime 2}+m_{q}^{2}}{\beta(1-\beta) Q^{2}}\right),
$$

and we have

$$
\frac{1}{z}=1+\frac{\kappa_{t}^{2}+m_{q}^{2}}{(1-\beta) Q^{2}}+\frac{k_{t}^{2}+\kappa_{t}^{2}-2 \kappa_{\mathbf{t}} \cdot \mathbf{k}_{\mathbf{t}}+m_{q}^{2}}{\beta Q^{2}} .
$$

As in Ref. [47], the scale $\mu$, which controls the unintegrated gluon and the QCD coupling constant $\alpha_{s}$, is chosen as follows:

$\mu^{2}=k_{t}^{2}+\kappa_{t}^{2}+m_{q}^{2}$.

One should note that the coefficients used for quark and nonperturbative gluon contributions depend on the transverse momentum. As has been briefly explained before, the main prescription for $F_{L}$ consists of three terms; the first term is the $k_{t}$-factorization which explains the contribution of the UPDF into the $F_{L}$. This term is derived with the use of a pure gluon contribution. However, it only counts the gluon contributions coming from the perturbative region, i.e., for $k_{t}>1 \mathrm{GeV}$, and does not have anything to do with the non- 
perturbative contributions. In Ref. [73], it has been shown that a proper non-perturbative term can be derived from the $k_{t}$ factorization term, making compact the $k_{t}$ dependence and the integration with the use of a variable-change, i.e., $y$, that carries the $k_{t}$ dependence. Nevertheless, there is a calculable quark contribution in the longitudinal structure function of the proton, which comes from the collinear factorization, i.e. the second term of Eq. (7).

For the charm quark, $m$ is taken to be $m_{c}=1.4 \mathrm{GeV}$, and $u, d$, and $s$ quark masses are neglected. We also use the same approximation to save the computation time [19], the one we did for the calculation of $F_{2}\left(x, Q^{2}\right)$ [32], i.e., the representative "average" value for $\phi,\langle\phi\rangle=\frac{\pi}{4}$ for the perturbative gluon contribution. This approximation has been checked in Ref. [19] (p. 83). The rest of the $\phi$ angular integration can be performed analytically by using a series of integral identities given in Ref. [75]. We will also verify this approximation in the next section. The unintegrated gluon distributions are not defined for $k_{t}$ and $\kappa_{t}<k_{0}$, i.e. the nonperturbative region. So, according to Ref. [70], $k_{0}$ is chosen to be about $1 \mathrm{GeV}$, which is around the charm mass in the present calculation, as it should. On the other hand, one expects that the discrepancy between the $k_{t}$-factorization calculation and the experimental data can be eliminated by using the PDFs, which have been fitted to the same data for $F_{2}\left(x, Q^{2}\right)$ [76] with respect to the re-summation method of KMS [47].

\section{Results, discussions, and conclusions}

In Fig. 2, the longitudinal proton structure functions in the frameworks of KMR (left panels) and MRW (right panels) formalisms, by using the MRST99 [52] and the MSTW2008NLO [53] PDF inputs, versus $\mathrm{x}$, for $Q^{2}=2,4,6,12$, and $15 \mathrm{GeV}^{2}$ are plotted, respectively. Their total $F_{L}\left(x, Q^{2}\right)$ and the contributions from the $k_{t}$-factorization scheme, the quark, and the non-perturbative parts (see Eq. (7) are presented with different curve styles. The behavior of $F_{L}\left(x, Q^{2}\right)$ mostly comes from the $k_{t}$-factorization contribution, especially as $Q^{2}$ is increased, and it is more sizable in the case of the MRW approach. By increasing the $Q^{2}$ values the contribution of the $k_{t}$-factorization becomes dominant. Another point is the decrease of the non-perturbative parts at small $x$, in the case of the MRW scheme. As we discussed in our previous works, this is expected. The KMR constraint spreads the UPDF to the whole transverse momentum region [32] and it sums up the both leading DGLAP and BFKL logarithms contributions. The general behavior of two schemes in Fig. 2 shows some differences also at lower $Q^{2}$ scales, while the values and behaviors of quarks and $k_{t}$ factorization portions in both formalisms are almost similar; the non-perturbative contributions have more different values and behavior for $x \simeq 0.01$. The latter point plays a main role in the discrepancies of the total $F_{L}\left(x, Q^{2}\right)$ at lower $Q^{2}$. On the other hand the non-perturbative contribution in each case remains almost fixed through the variation of $Q^{2}$. These effects have their roots in the parent PDF sets at non-perturbative boundary which is very sensitive to the discipline and procedure of the PDF generating group. This figure can also be compared with Fig. 2 of GS [45] at $Q^{2}=2,4$, and $6 \mathrm{GeV}^{2}$. There is general agreement between our approaches and those of GS, which have used the DGLAP/BFKL re-summation method, developed by Kwiecinski, Martin and Stasto (KMS) [47], for a calculation of the unintegrated gluon density at small $x$. This agreement is more visible at larger $Q^{2}$ and in the KMR approach, which is expected. However, our longitudinal proton structure function results go smoothly to zero with respect to those of GS as $x$ becomes larger. The reason is both our input PDF, which is valid for the whole $\left(x, Q^{2}\right)$ plane, and the calculation of the UPDFs which are calculated by using the KMR and MRW approaches, which are to fulfill the DGLAP requirements.

Our longitudinal proton structure function results for larger values of $Q^{2}$, with the different input PDFs, i.e., MERST99 [52], MSTW2008-LO (using KMR formalism) and MSTW2008-NLO [53] (using MRW formalism) are given in Figs. 3, 4, and 5, respectively. Again the total $F_{L}\left(x, Q^{2}\right)$ and the contributions from the $k_{t}$-factorization scheme, the quarks, and the non-perturbative parts are presented with different curve styles. The results are a mostly decreasing function $\mathrm{x}$, for the various values of $Q^{2}$. There are sizable differences between the MERST99 and MSTW2008-LO. On the other hand, as one should expect, for a large value of $Q^{2}$ the results of KMR and MRW behave more similarly. As we pointed out before, again the $k_{t}$-factorization contributions are dominant. The increase in the values of $F_{L}\left(x, Q^{2}\right)$ in Fig. 4 is due to the increase of the input PDF at LO approximation. The reason is that the results of $F_{L}\left(x, Q^{2}\right)$ approach the same values as $x$ and $Q^{2}$ increases, which is a heritage of the parent DGLAP evolution.

In order to analyze the above $Q^{2}$ dependent more clearly, in Fig. 6, the longitudinal proton structure functions are plotted against $Q^{2}$ for the two different values of $x=0.001$ and 0.0001 . Note that for large $Q^{2}$, especially the MRW approach needs a large computation time. So we have stopped at $Q^{2}=100 \mathrm{GeV}^{2}$ for this procedure. There are sizable differences between the two approaches and results coming from the two different input PDFs. But this should not be very important regarding the experimental data that we will discuss later on. In Fig. 7, a comparison is made between the three different $F_{L}\left(x, Q^{2}\right)$ results, namely the KMR procedure with MERST99 and MSTW2008-LO inputs and MRW scheme with MSTW2008-NLO inputs. Especially 
Fig. 2 The longitudinal proton structure functions in the frameworks of KMR (left panels, using the MRST99 PDF data as inputs) and MRW (right panels, using the

MSTW2008-NLO data as inputs) UPDF, versus $\mathrm{x}$, for $Q^{2}=2,4,6,12$, and $15 \mathrm{GeV}^{2}$.

Their total value and the contributions of the $k_{t}$ factorization scheme, the quark, and the non-perturbative parts are presented with different curve styles
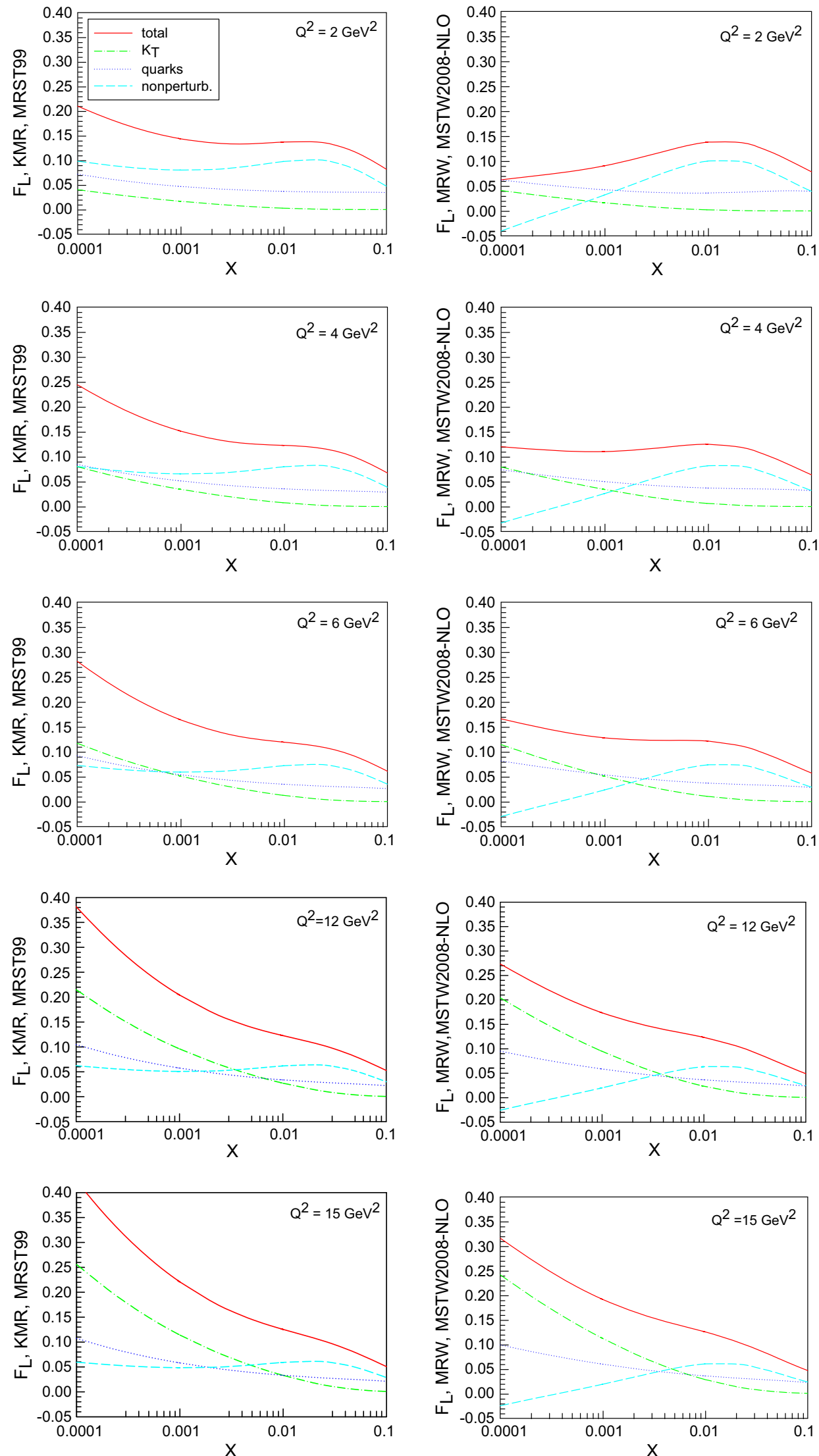
Fig. 3 The longitudinal proton structure functions in the frameworks of KMR by using the MRST99 PDF data versus $x$, for $Q^{2}=12,15,20,25$,

$35,45,60,80,90$, and 110 $\mathrm{GeV}^{2}$. Their total value and the contributions of the

$k_{t}$-factorization scheme, the quark, and the non-perturbative parts are presented with different curve styles
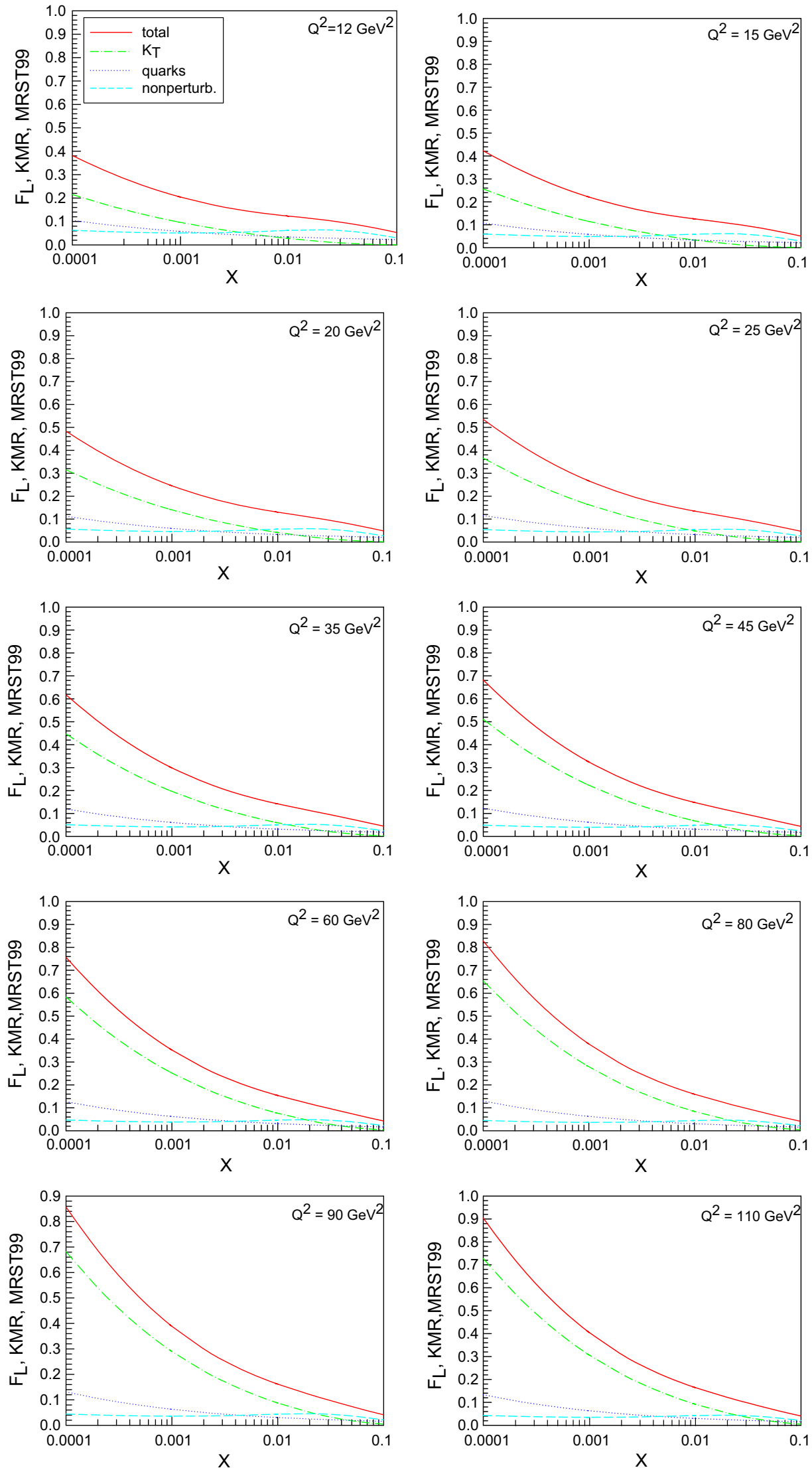
Fig. 4 The longitudinal proton structure functions in the frameworks of KMR by using the MSTW2008-LO PDF data versus $\mathrm{x}$, for $Q^{2}=$

$12,15,20,25,35,45,60,80,90$, and $110 \mathrm{GeV}^{2}$. Their total value and the contributions of the $k_{t}$-factorization scheme, the quark, and the non-perturbative parts are presented with different curve styles
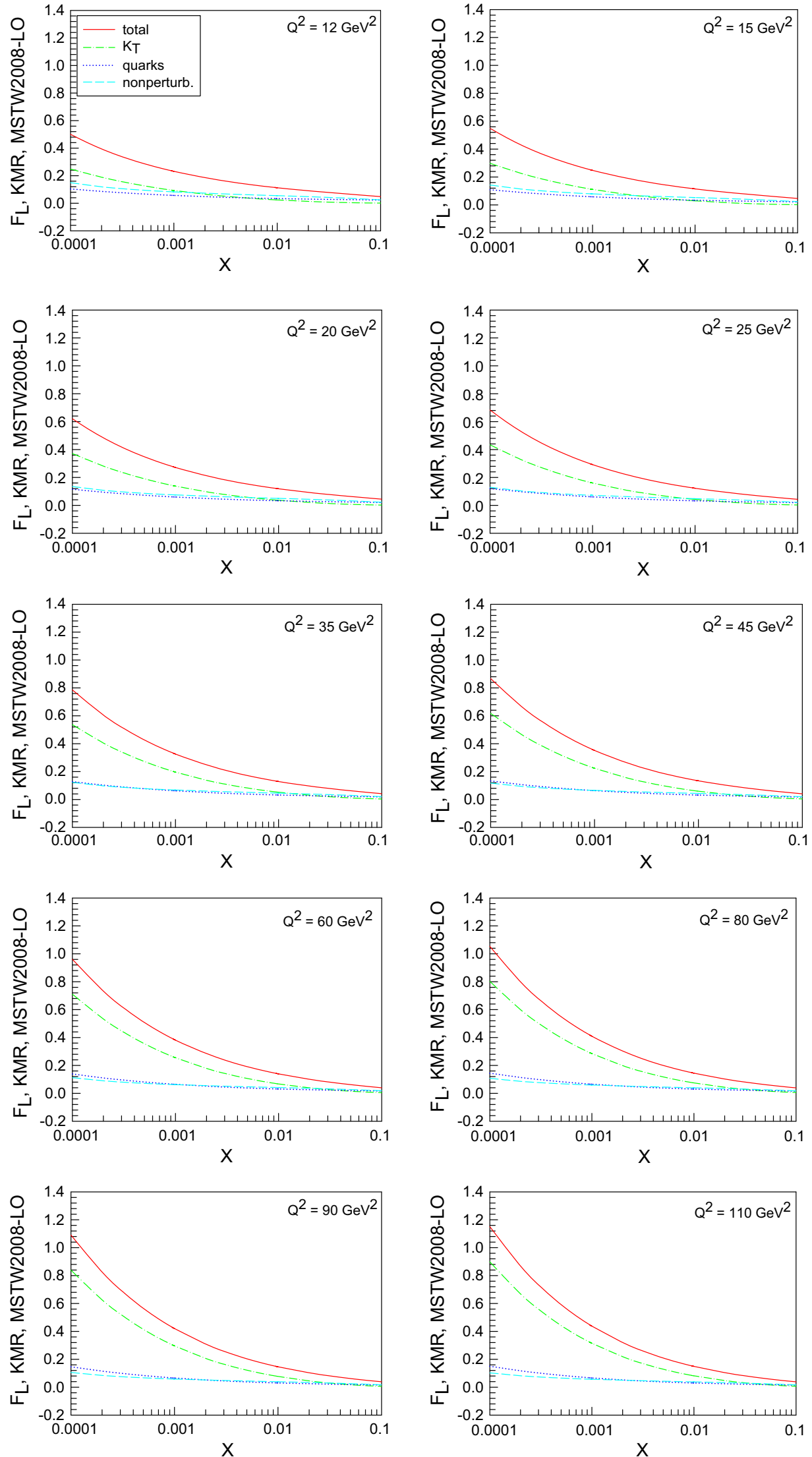

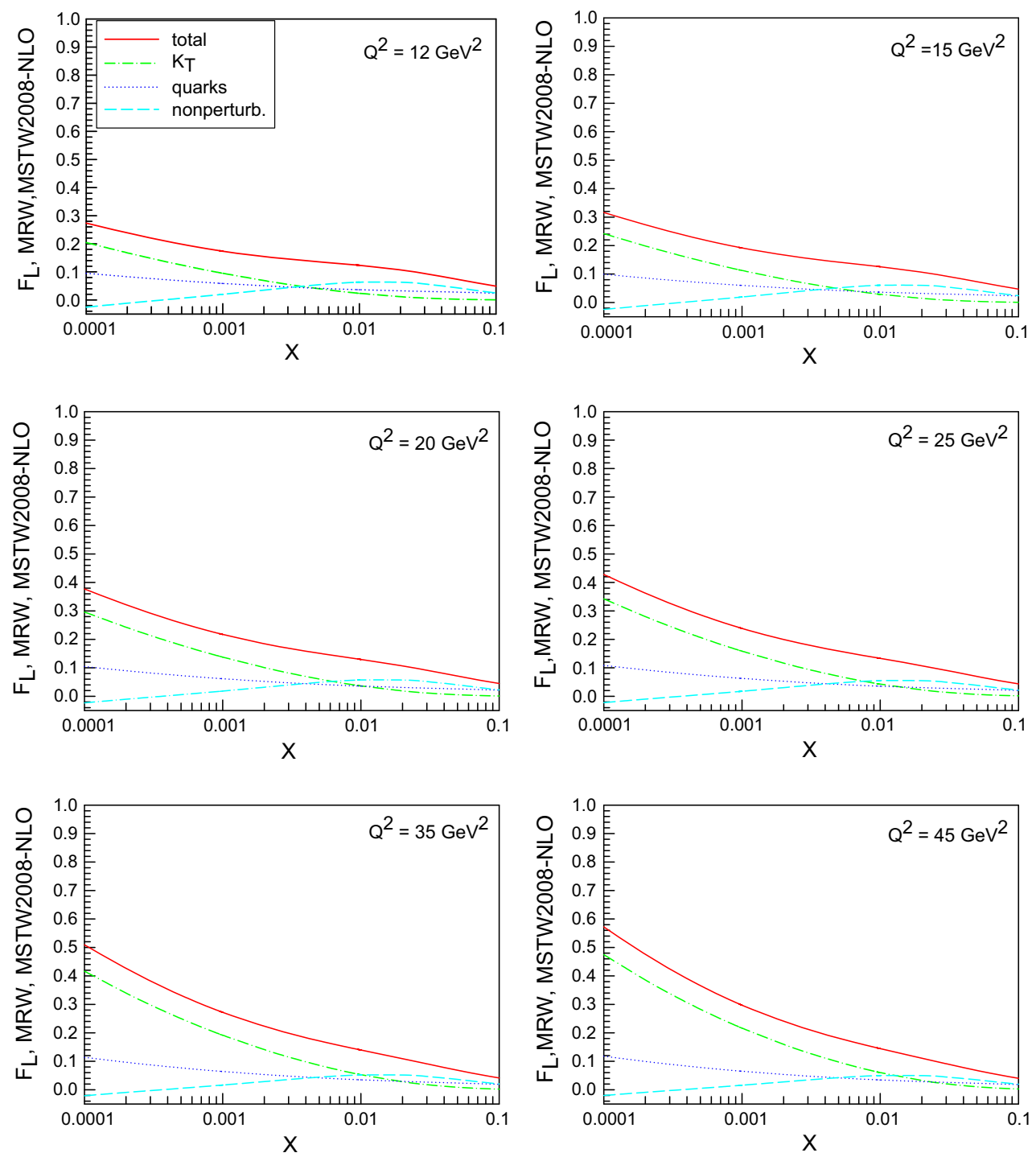

Fig. 5 The longitudinal proton structure functions in the frameworks of MRW and by using the MSTW2008-NLO PDF data versus $x$ for $Q^{2}=12,15,20,25,35$, and $45 \mathrm{GeV}^{2}$. Their total value and the

contributions of the $k_{t}$-factorization scheme, the quark, and the nonperturbative parts are presented with different curve styles

there are large differences between the KMR and MRW approaches at large $Q^{2}$. The above results can be directly compared to that of GS [45] (see their Fig. 3). A very similar behavior is observed especially between the $k_{t}$-factorization approaches.

In Figs. 8, 9, and 10, we present our results in the range of energy available in the $\mathrm{H} 1$ and ZEUS data [31], respectively. Note that for $Q^{2} \geq 80 \mathrm{GeV}^{2}$, because of the large computation time, we have only given four points (filled squares) for the MRW case. Very good agreement is observed between our result and those of the experimental data at different $Q^{2}$ and $x$ values. It seems that with present existing data as regards the UPDFs of gluons generated with different input PDF and constraint procedures, one can reasonably explain the $\mathrm{H} 1$ and the ZEUS experimental data. It seems that even at

low energies and small $x$ values (see Fig. 8), we find good agreement between our calculation and the available data. However, as we mentioned before and has been stated by several authors, the $F_{L}$ is mainly driven through the gluons distributions, especially at low values of $x$. The fact that $F_{2}$ is not accurately fit to the data (see our previous work [32]), though we get good agreement between the $F_{L}$ calculations and $\mathrm{H} 1$ and ZEUS data, could be caused of the quark-quark contributions, leading to a higher contribution to $F_{2}$. Since $F_{L}$ is more sensitive to the gluons' UPDFs with respect to $F_{2}$, one can conclude that the present calculation might confirm that the KMR and MRW procedures (for generating the gluon UPDFs) and the $k_{t}$-factorization scheme can reproduce a reasonable $F_{2}$ (considering our previous work [32]) and present $F_{L}$. On the other hand, as we stated previously: 

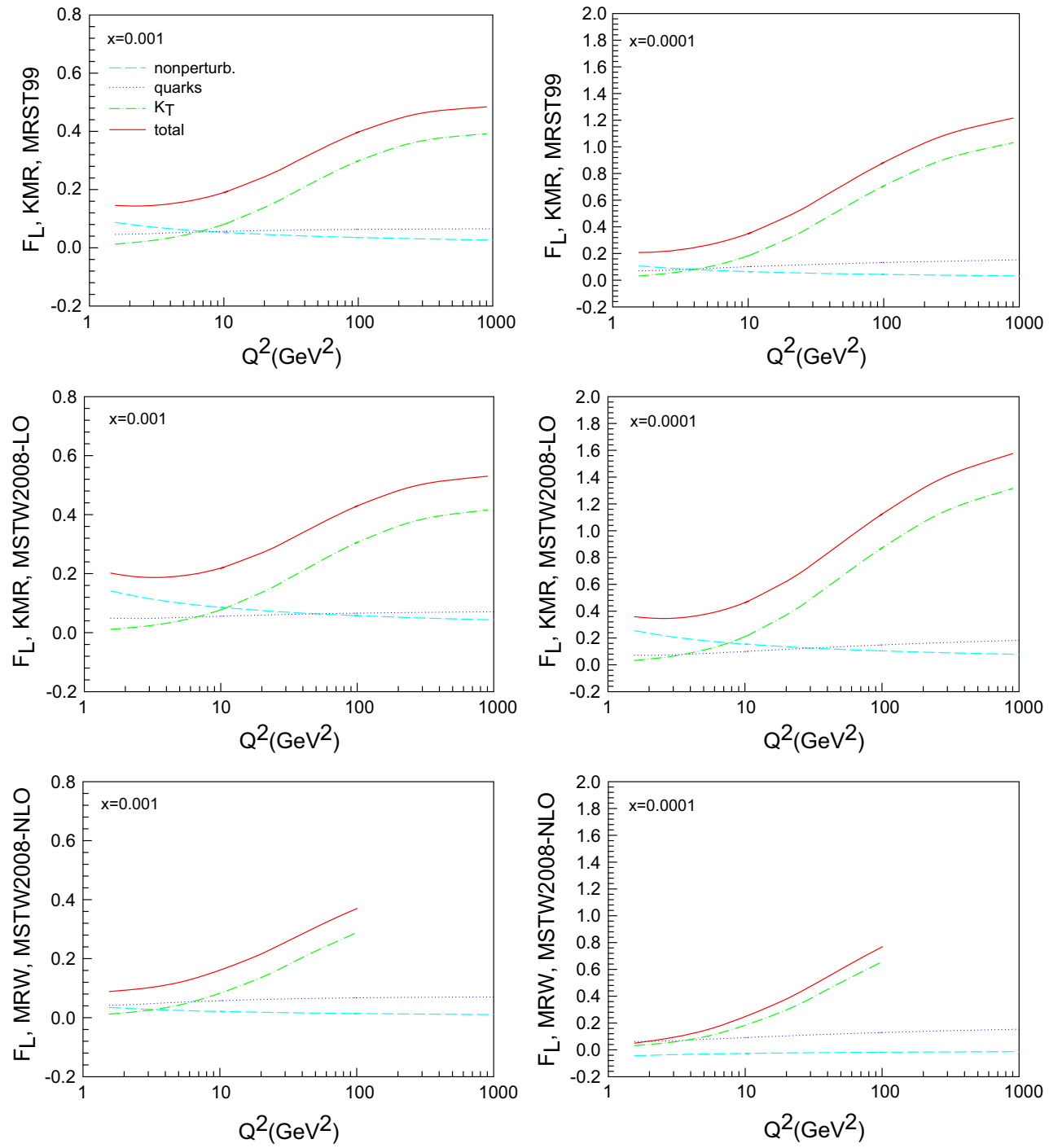

Fig. 6 The longitudinal proton structure functions in the frameworks of KMR and MRW by using the MRST99, MSTW2008-LO and MSTW2008-NLO PDF data versus $Q^{2}\left(\mathrm{GeV}^{2}\right)$, for fix $x=0.001$ and

0.0001. Their total values and the contributions of the $k_{t}$-factorization scheme, the quark, and the non-perturbative parts are presented with different curve styles
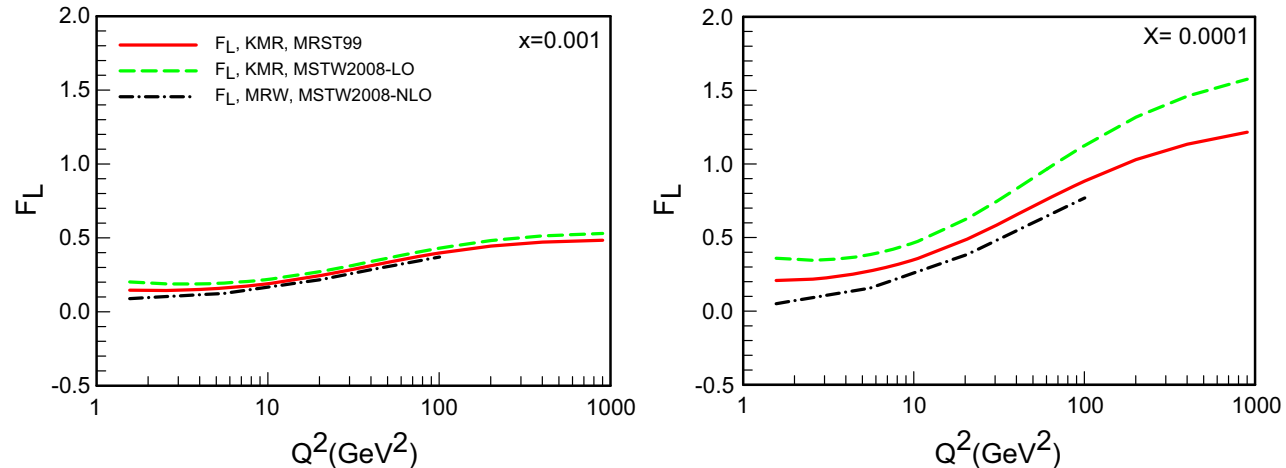

Fig. 7 The comparison of the total longitudinal proton structure functions in the frameworks of KMR and MRW by using the MRST99, MSTW2008LO and MSTW2008-NLO PDF data versus $Q^{2}\left(\mathrm{GeV}^{2}\right)$, for the fixed values $x=0.001$ and 0.0001 

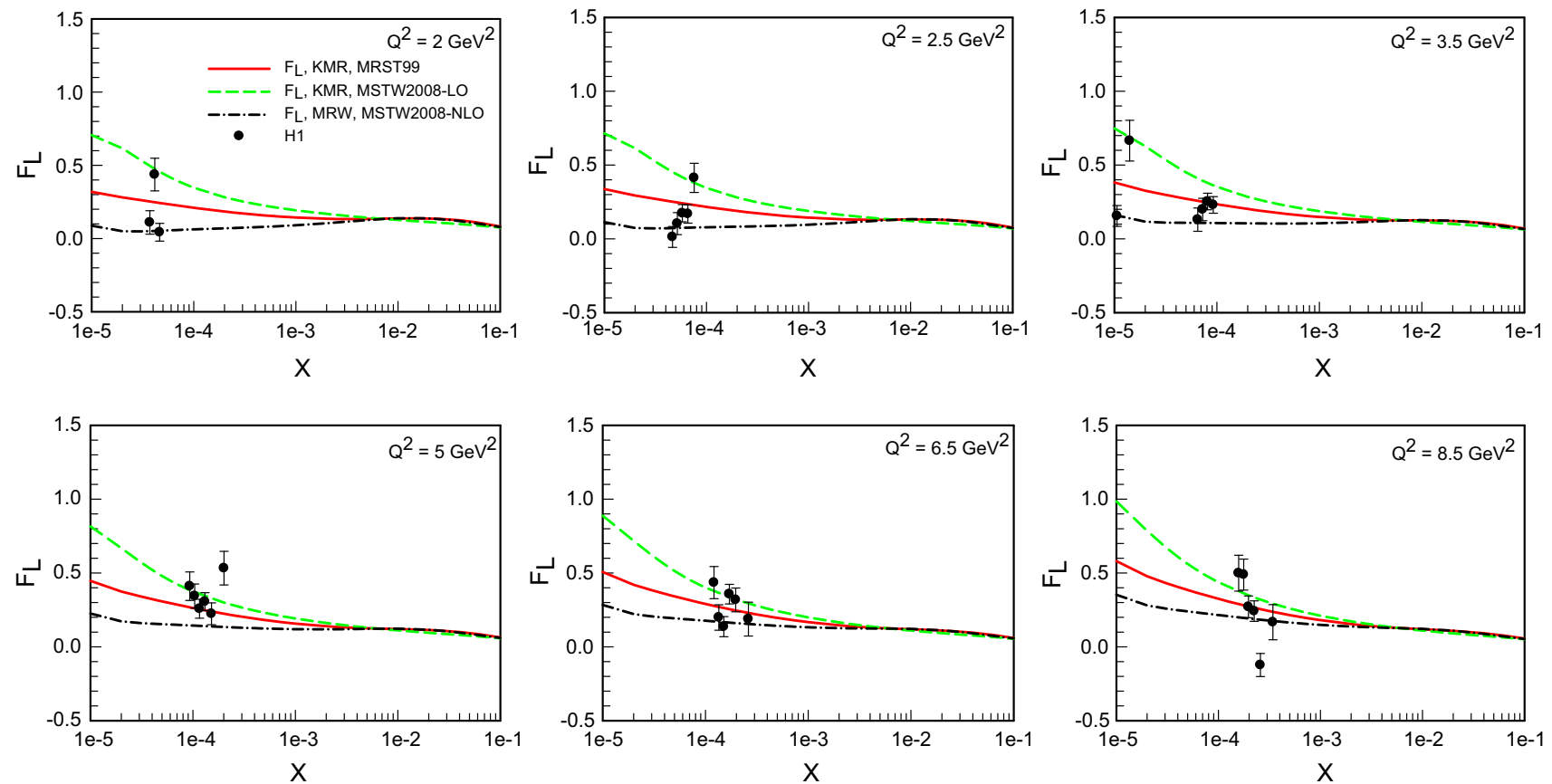

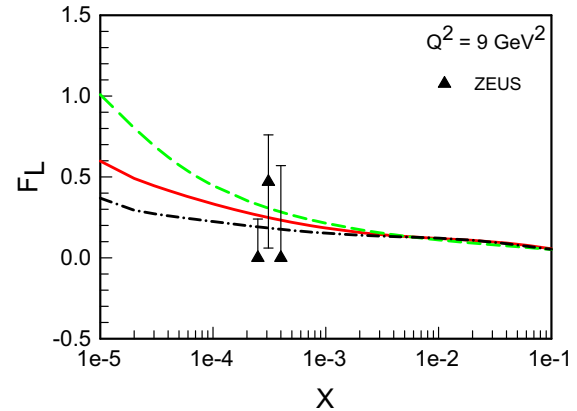

Fig. 8 The comparison of the total longitudinal proton structure functions, in the frameworks of KMR and MRW by using the MRST99, MSTW2008-LO, and MSTW2008-NLO PDF data versus $x$ at $Q^{2}=$

(1) present results also show good agreement with the theoretical calculations of GS, which have used a more complicated approach such as KMS; (2) it is interesting that the KMR and MRW UPDF can generate reasonable $F_{L}$ without using any free parameter in the $\left(x, Q^{2}\right)$ plane even at low $Q^{2}$ (regarding Fig. 8), especially the UPDF generated for the gluons.

Finally, the verification of the fact that the $\phi$ integration of the perturbative gluon contribution can be averaged by setting $\langle\phi\rangle=\pi / 4$, which was discussed in the end of previous section, is presented in Fig. 11, for four values of $Q^{2}=3.5,12,60$, and $110 \mathrm{Gev}^{2}$ by using the KMR formalism and the MRST99. It is clearly seen that the above approximation does work properly and one can save much computation time. It should be pointed out that only the upper cut and the factorization scale will be affected by the $\phi$ approximation.
2, 2.5, 3.5, 5, 6.5, 8.5 and $9 \mathrm{GeV}^{2}$, with the corresponding ZEUS and H1 data (filled triangles and bold points), respectively

In conclusion, the longitudinal proton structure functions, $F_{L}\left(x, Q^{2}\right)$, were calculated based on the $k_{t}$-factorization formalism, by using the UPDF which are generated through the KMR and MRW procedures. The LO UPDF of the KMR prescription is extracted, by taking into account the PDFs of MSTW2008-LO and MRST99-NLO, and also the NLO UPDF of the MRW scheme is generated through the set of MSTW2008-NLO PDFs as the inputs. The different aspects of the $F_{L}\left(x, Q^{2}\right)$ in the two approaches, as well as its perturbative and non-perturbative parts, were calculated and discussed. It was shown that our approaches are in agreement with those given by GS. Then the comparison of $F_{L}\left(x, Q^{2}\right)$ was made with the data given by the ZEUS and H1 collaborations at HERA. It was demonstrated that the extracted longitudinal proton structure functions based on the UPDFs of the above two schemes were consistent with the experimental data, and by a good approximation, they are inde- 

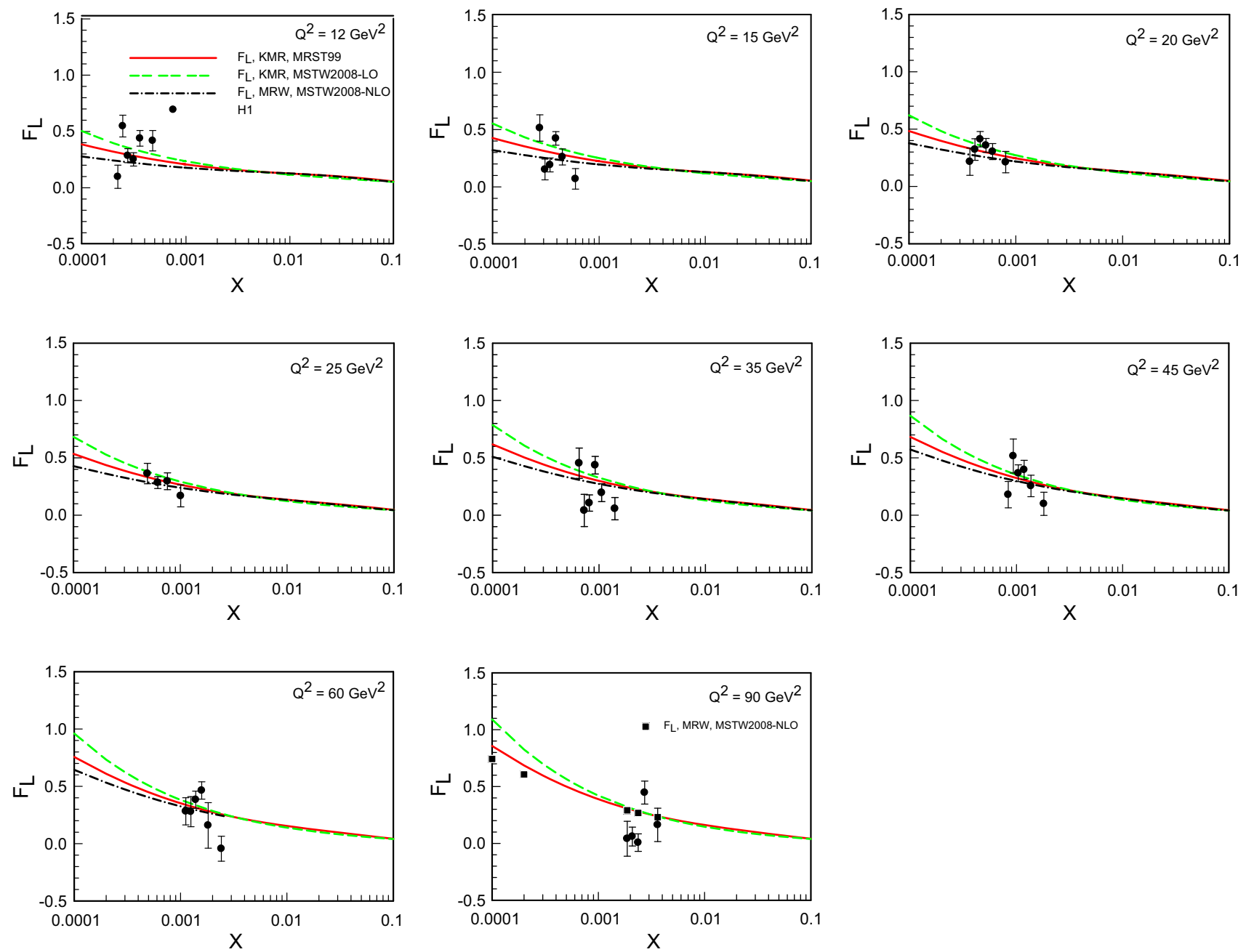

Fig. 9 The comparison of total longitudinal proton structure functions, in the frameworks of KMR and MRW by using the MRST99, MSTW2008LO, and MSTW2008-NLO PDF data versus $x$ at $Q^{2}=12,15,20,25,35,45,60$, and $90 \mathrm{GeV}^{2}$, with the corresponding $\mathrm{H} 1$ data (bold points)

pendent to the input PDF. But as was pointed out in our previous work [32], the one developed from the KMR prescription has better agreement with the data with respect to that of MRW. Although the MRW formalism is more in compliance with the DGLAP evolution equations requisites, it seems, in the KMR case, that the angular ordering constraint spreads the UPDF to whole transverse momentum region, and it makes the results sum up the leading DGLAP and BFKL logarithms. At first, it seems that there should be theoretical support for applying the angular ordering condition only to the diagonal splitting functions, in accordance with Ref. [22]. But as has been mentioned in Refs. [32,33], these phenomenological modifications of the KMR approach (including the application of the AOC to all splitting functions) work as an "effective model" that spreads the UPDFs to the region $k_{t}>\mu$ (a characteristic of low $x$ physics), representing a good level of agreement with the data. Beside this, in our new work [33], in which we have calculated the $F_{L}$ in the dipole approximation according to the LO prescription of Ref. [22], it is shown that there is not much difference if one applies the AOC to all the splitting functions, i.e., one uses the KMR UPDF instead of using the LO prescription of Ref. [22]. On the other hand, in this paper we have focused ona comparison of the LO and the NLO calculation of $F_{L}$, and since the calculations are very time consuming, we restricted the results to the LO-KMR and NLO-MRW. We should also point out here that there exists a formalism called the "reggeized quark" formalism [77-79], in which the $K_{T}$ dependence of PDFs are treated on the same footing as the one we did for the gluons. However, at small $x$ region the gluon channel is dominant and our approximation is acceptable.

As has been suggested in Ref. [45], by lowering the factorization scale or the Bjorken variable in the experimental measurements, it may be possible to analyze the present theoretical approaches more accurately. 

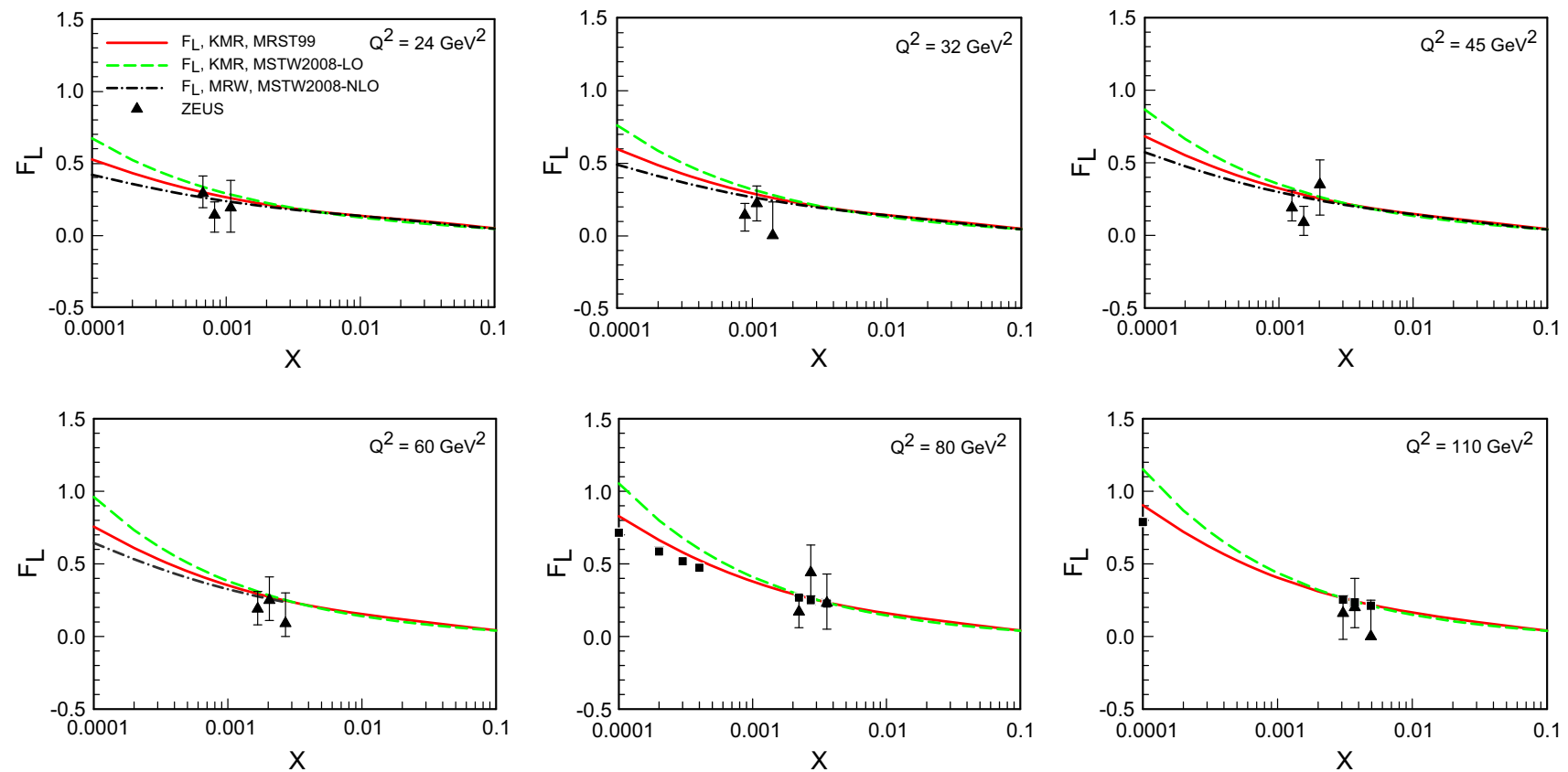

Fig. 10 The comparison of total longitudinal proton structure functions, in the frameworks of KMR and MRW by using the MRST99, MSTW2008-LO, and MSTW2008-NLO PDF data versus $x$ at $Q^{2}=$

24, 32, 45, 80, and $110 \mathrm{GeV}^{2}$, with the corresponding ZEUS and $\mathrm{H} 1$ data (filled triangle and bold points), respectively
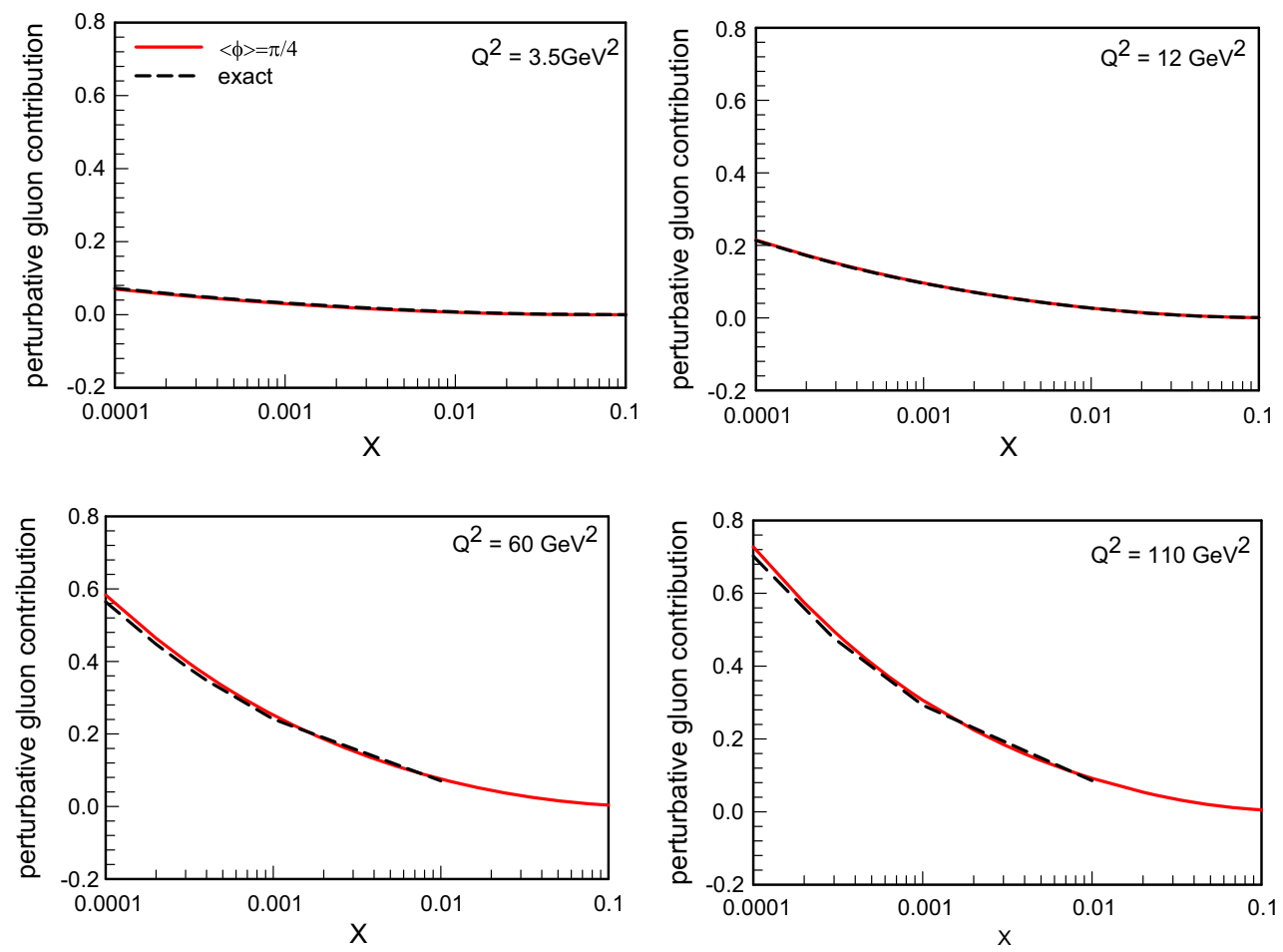

Fig. 11 The comparison of perturbative gluon contribution to $F_{L}$ by performing the $\phi$ integration (exact) and the approximated one with $\phi=\pi / 4$, in the frameworks of KMR by using the MRST99 versus $x$ at $Q^{2}=3.5,12,60$, and $110 \mathrm{GeV}^{2}$

Acknowledgments MM would like to acknowledge the Research Council of University of Tehran and Institute for Research and Planning in Higher Education for the grants provided.
Open Access This article is distributed under the terms of the Creative Commons Attribution 4.0 International License (http://creativecomm ons.org/licenses/by/4.0/), which permits unrestricted use, distribution, 
and reproduction in any medium, provided you give appropriate credit to the original author(s) and the source, provide a link to the Creative Commons license, and indicate if changes were made. Funded by SCOAP ${ }^{3}$.

\section{References}

1. M. Ciafaloni, Nucl. Phys. B 296, 49 (1988)

2. S. Catani, F. Fiorani, G. Marchesini, Phys. Lett. B 234, 339 (1990)

3. S. Catani, F. Fiorani, G. Marchesini, Nucl. Phys. B 336, 18 (1990)

4. G. Marchesini, in Proceedings of the Workshop QCD at $200 \mathrm{TeV}$ Erice, Italy, ed. by L. Cifarelli, Yu.L. Dokshitzer, vol. 183. Plenum, New York (1992)

5. G. Marchesini, Nucl. Phys. B 445, 49 (1995)

6. V.N. Gribov, L.N. Lipatov, Yad. Fiz. 15, 781 (1972)

7. L.N. Lipatov, Sov. J. Nucl. Phys. 20, 94 (1975)

8. G. Altarelli, G. Parisi, Nucl. Phys. B 126, 298 (1977)

9. Y.L. Dokshitzer, Sov. Phys. JETP 46, 641 (1977)

10. H. Kharraziha, L. Lönnblad, JHEP 03, 006 (1998)

11. G. Marchesini, B. Webber, Nucl. Phys. B 349, 617 (1991)

12. G. Marchesini, B. Webber, Nucl. Phys. B 386, 215 (1992)

13. H. Jung, Nucl. Phys. B 79, 429 (1999)

14. H. Jung, G.P. Salam, Eur. Phys. J. C 19, 351 (2001)

15. H Jung, J. Phys. G Nucl. Part. Phys. 28, 971 (2002)

16. H. Jung et al., Eur. Phys. J. C 70, 1237 (2010)

17. H. Jung, M. Kraemer, A.V. Lipatov, N.P. Zotov, JHEP 01, 085 (2011)

18. M.A. Kimber, A.D. Martin, M.G. Ryskin, Phys. Rev. D 63, 114027 (2001)

19. M.A. Kimber, Unintegrated Parton Distributions, Ph.D. Thesis, University of Durham, UK (2001)

20. A.D. Martin, M.G. Ryskin, G. Watt, Eur. Phys. J. C 66, 163 (2010)

21. G. Watt, Parton Distributions. Ph.D. Thesis, University of Durham, UK (2004)

22. G. Watt, A.D. Martin, M.G. Ryskin, Eur. Phys. J. C 31, 73 (2003)

23. G. Watt, A.D. Martin, M.G. Ryskin, Phys. Rev. D 70, 014012 (2004)

24. M. Modarres, H. Hosseinkhani, N. Olanj, Nucl. Phys. A 902, 21 (2013)

25. M. Modarres, H. Hosseinkhani, Few-Body Syst. 47, 237 (2010)

26. M. Modarres, H. Hosseinkhani, Nucl. Phys. A 815, 40 (2009)

27. H. Hosseinkhani, M. Modarres, Phys. Lett. B 694, 355 (2011)

28. H. Hosseinkhani, M. Modarres, Phys. Lett. B 708, 75 (2012)

29. NMC: Arneodo et al., Nucl. Phys. B 483, 3 (1997)

30. ZEUS: Derrick et al., Zeit. Phys. C 72, 399 (1996)

31. H1 and ZEUS collaborations, JHEP 01, 109 (2010)

32. M. Modarres, H. Hosseinkhani, N. Olanj, Phys. Rev. D 89, 034015 (2014)

33. M. Modarres, M.R. Masouminia, H. Hosseinkhani, N. Olanj (2015) (submitted for publication)

34. V.S. Fadin, E.A. Kuraev, L.N. Lipatov, Phys. Lett. B 60, 50 (1975)

35. L.N. Lipatov, Sov. J. Nucl. Phys. 23, 642 (1976)

36. E.A. Kuraev, L.N. Lipatov, V.S. Fadin, Sov. Phys. JETP 44, 45 (1976)

37. E.A. Kuraev, L.N. Lipatov, V.S. Fadin, Sov. Phys. JETP 45, 199 (1977)

38. Ya.Ya. Balitsky, L.N. Lipatov, Sov. J. Nucl. Phys. 28, 822 (1978)

39. S. Catani, M. Ciafaloni, F. Hautmann, Phys. Lett. B 242, 97 (1990)

40. S. Catani, M. Ciafaloni, F. Hautmann, Nucl. Phys. B 366, 135 (1991)

41. J.C. Collins, R.K. Ellis, Nucl. Phys. B 360, 3 (1991)

42. E.M. Levin, M.G. Ryskin, YuM Shabelski, A.G. Shuvaev, Sov. J. Nucl. Phys. 54, 867 (1991)
43. J. Kwiecinski, A.D. Martin, A.M. Stasto, Acta Physica Polonica B 28, 2577 (1997)

44. R.G. Robersts, The Structure of the Proton. Cambridge University Press, Cambridge (1993)

45. K. Golec-Biernat, A.M. Stasto, Phys. Rev. D 80, 014006 (2009)

46. A.M. Stasto, Phys. Lett. B 679, 288 (2009)

47. J. Kwiecinski, A.D. Martin, A.M. Stasto, Phys. Rev. D 56, 3991 (1997)

48. F.D. Aaron et al (H1 Collaboration), Phys. Lett. B 665, 139 (2008)

49. V. Andreev et al., H1 Collaboration, Eur. Phys. J. C 74, 2814 (2014)

50. ZEUS collaboration. Desy Report No. Desy-09-045 (2009)

51. ZEUS collaboration, Phys. Rev. D 90, 072002 (2014)

52. A.D. Martin, R.G. Roberts, W.J. Stirling, R.S. Thorne, Eur. Phys. J. C 14, 133 (2000)

53. A.D. Martin, W.J. Stirling, R.S. Thorne, G. Watt, Eur. Phys. J. C 63, 189 (2009)

54. G. Marchesini, B.R. Webber, Nucl. Phys. B 310, 461 (1988)

55. YuL Dokshitzer, V.A. Khoze, S.I. Troyan, A.H. Mueller, Rev. Mod. Phys. 60, 373 (1988)

56. V.A. Saleev, Phys. Rev. D 80, 114016 (2009)

57. A.V. Lipatov, N.P. Zotov, Phys. Rev. D 81, 094027 (2010)

58. S.P. Baranov, A.V. Lipatov, N.P. Zotov, Phys. Rev. D 81, 094034 (2010)

59. B.A. Kniehl, V.A. Saleev, A.V. Shipilova, Phys. Rev. D 81, 094010 (2010)

60. S.P. Baranov, A.V. Lipatov, N.P. Zotov, Eur. Phys. J. C 71, 1631 (2011)

61. A.V. Lipatov, M.A. Malyshev, N.P. Zotov, Phys. Lett. B 699, 93 (2011)

62. H. Jung, M. Kraemer, A.V. Lipatov, N.P. Zotov. arXiv:1105.5071 [hep-ph] (2011)

63. H. Jung, M. Kraemer, A.V. Lipatov, N.P. Zotov, Phys. Rev. D 85, 034035 (2012)

64. A.V. Lipatov, N.P. Zotov, Phys. Lett. B 704, 189 (2011)

65. B.A. Kniehl, V.A. Saleev, A.V. Shipilova, E.V. Yatsenko (2011). arXiv:1107.1462 [hep-ph]

66. H. Jung, M. Kraemer, A.V. Lipatov, N.P. Zotov, Proceedings of 19th international workshop on deep-inelastic scattering and related subjects (DIS 2011). arXiv:1107.4328 [hep-ph] (2011)

67. W. Furmanski, R. Petronzio, Phys. Lett. B 97, 437 (1980)

68. S. Catani, F. Hautmann, Nucl. Phys. B 427, 745 (1994)

69. M. Ciafaloni, Phys. Lett. B 356, 437 (1995)

70. A.J. Askew, J. Kwiecinski, A.D. Martin, P.J. Sutton, Phys. Rev. D 47, $3775(1993)$

71. A.J. Askew, J. Kwiecinski, A.D. Martin, P.J. Sutton, Phys. Rev. D 49, 4402 (1994)

72. A.J. Askew, Small x Physics, Thesis Presented for the Degree of Doctor of Philosophy, University of Durham (1995)

73. A.M. Stasto, Acta. Phys. Polo. B 27, 1353 (1996)

74. A.M. Stasto, QCD Analysis of Deep Inelestic Lepton-Hadron Scattering in the Region of Small Values of the Bjorken Parameter, Thesis Presented for the Degree of Doctor of Philosophy, University of Durham (1999)

75. I.S. Gradshteyn, I.M. Ryzhik, Table of Integrals, Series, and Products, Corrected and Enlarged Edition. Academic Press, New York (1980)

76. M.A. Kimber, J. Kwiecinski, A.D. Martin, A.M. Stasto, Phys. Rev. D 62, 094006 (2000)

77. B.A. Kniehl, M.A. Nefedov, V.A. Saleev, Phys. Rev. D 89, 114016 (2014)

78. S.P. Baranov, A.V. Lipatov, N.P. Zotov, Phys. Rev. D 89, 094025 (2014)

79. F. Hautmann, M. Hentschinski, H. Jung, Nucl. Phys. B 865, 54-66 (2012) 